\title{
Fibroma Odontogénico Central de Mandíbula: Revisión de la Literatura a Propósito de un Caso
}

\section{Central Odontogenic Fibroma of the Mandible: A Short Literature Review Apropos of a Case}

\author{
Edgard Riquelme-Medel ${ }^{1 *}$, Sebastián Alegría-Muñoz ${ }^{2}$, Rodrigo Badilla-Monasterio ${ }^{2}$
}

1. Cirujano Dentista, Pasante Servicio de Cirugía Oral y Maxilofacial, Hospital Clínico Félix Bulnes Cerda, Santiago, Chile.

2. Cirujano Dentista, Especialista en Cirugía Oral y Maxilofacial, Hospital Clínico Félix Bulnes Cerda, Santiago, Chile.

${ }^{*}$ Correspondencia a: RodrigoBadilla-Monasterio | E-mail: badillamonasterio@gmail.com Trabajo recibido el 14/05/2019.

Aprobado para su publicación el 19/09/2019

\begin{abstract}
RESUMEN
El fibroma odontogénico central (FOC) es una neoplasia benigna poco frecuente que representa alrededor del $1.5 \%$ de los tumores odontogénicos intraóseos. Su presentación es exclusiva de huesos maxilares. Tiene origen mesodérmico, pudiendo derivar del folículo dentario, ligamento periodontal y/o la papila dental. Tradicionalmente presenta dos variantes histológicas: un tipo que es pobre en epitelio y otro tipo que es rico en epitelio con focos de material calcificado. En la mayoría de los casos muestra un crecimiento lento y progresivo que produce expansión cortical con o sin sintomatología. Radiográficamente es común observar una imagen radiolúcida y unilocular, raramente se pueden exhibir lesiones multiloculares y/o de radiolucidez mixta. El tratamiento indicado en la mayoría de los casos es la enucleación del tumor. El siguiente reporte de caso describe las características clínicas, imageneológicas e histopatológicas de una lesión mandibular con diagnóstico de FOC y cuyo tratamiento realizado fue la enucleación del tumor.
\end{abstract}

\section{PALABRAS CLAVE}

Fibroma odontogénico central; Tumores odontogénicos; Tumores maxilares.

Rev. Clin. Periodoncia Implantol. Rehabil. Oral Vol. 12(3); 144-147, 2019.

\section{ABSTRACT}

Central odontogenic fibroma (COF) is a rare benign neoplasm that accounts for about $1.5 \%$ of intraosseous odontogenic tumors. Its presentation is exclusively in the maxillary bones. It has a mesodermal origin, being able to derive from the dental follicle, periodontal ligament and/or the dental papilla. Traditionally, it has two histological variants: one type that is poor in epithelium and another type that is rich in epithelium with foci of calcified material. In most cases it shows a slow and progressive growth that produces cortical expansion with or without symptomatology. Radiographically, it is common to observe a radiolucent and unilocular image, but rarely multilocular lesions and / or mixed radiolucency can be exhibited. The treatment indicated in most cases is the enucleation of the tumor. The following case report describes the clinical, imaging and histopathological characteristics of a mandibular lesion diagnosed with FOC and whose treatment was enucleation of the tumor.

\section{KEYWORDS}

Central odontogenic fibroma; Odontogenic tumors, Maxillary tumors.

Rev. Clin. Periodoncia Implantol. Rehabil. Oral Vol. 12(3); 144-147, 2019.

\section{INTRODUCCIÓN}

El fibroma odontogénico central (FOC) es un tumor benigno intraóseo de origen odontogénico poco frecuente, considerado por la Organización Mundial de la Salud como una proliferación benigna del ectomesénquima caracterizada por tejido fibroblástico con una cantidad variable de epitelio odontogénico aparentemente inactivo ${ }^{(1)}$.

EI FOC abarca alrededor del 1,5\% de los tumores odontogénicos intraóseos ${ }^{(2)}$. Se presenta en pacientes entre los 11 a 80 años con una edad media de 34 años, distribuidos ampliamente entre la segunda y la sexta década. Muestra un predominio mayor por el género femenino $(2,8: 1)$ sobre el masculino. Muestra un crecimiento lento y progresivo que produce expansión cortical con o sin sintomatología ${ }^{(3)}$. La lesión se produce con mayor frecuencia en la mandíbula con respecto a maxilar $(6,5: 1)$ y se localiza preferentemente en la zona posterior de la mandíbula y en la zona anterior del maxilar ${ }^{(4)}$.

Histológicamente existen dos variantes principales: A) El tipo simple, que contiene escaso epitelio odontogénico inactivo con finas fibras colágenas dispersas y pequeños focos de calcificaciones distróficas. B) El tipo complejo (tipo WHO), compuesto de tejido conectivo celular y fibroblástico maduro, con islotes y cordones de epitelio odontogénico, y calcificaciones compuestas generalmente de material dentinario(5)

Las características radiográficas del tumor son variables; la mayoría de las lesiones son radiolúcidas y de bordes bien definidos. En menos casos se observan bordes escleróticos o de radiolucidez mixta. Pueden presentarse imágenes uni o multiloculares, y las lesiones de mayor tamaño tienden a ser multiloculares. También puede estar asociada a dientes no erupcionados, observarse desplazamiento y/o reabsorción de dientes adyacentes ${ }^{(6)}$.

El tratamiento quirúrgico del FOC es conservador, consistiendo en la enucleación de la lesión y legrado de la cavidad ósea remanente. Su recurrencia se considera infrecuente en el contexto de las recidivas, sin embargo, se debe considerar un seguimiento del paciente durante al menos 5 años ${ }^{(4)}$.

Se reporta un caso clínico de FOC, describiendo sus características clínicas, imageneológicas, histopatológicas y su tratamiento quirúrgico. 


\section{INFORME DE CASO}

Mujer de 29 años de edad, sin antecedentes mórbidos de relevancia, consulta en el Servicio de Cirugía Oral y Maxilofacial del Hospital Clínico Félix Bulnes Cerda de Santiago de Chile, por presentar aumento de volumen mandibular posterior derecho, sintomático, de 4 años de evolución. El examen clínico facial reveló una asimetría hemifacial derecha con un moderado aumento de volumen perimandibular (Fig. 1). Al examen clínico bucal se observó una lesión tumoral de gran tamaño que invadía vestíbulo bucal superior e inferior y que abarcaba las regiones de cuerpo, ángulo y rama mandibular derecha; de límites definidos, consistencia firme, asociada a dientes desplazados y recubierto por mucosa fibrosa como consecuencia de trauma oclusal (Fig. 2)

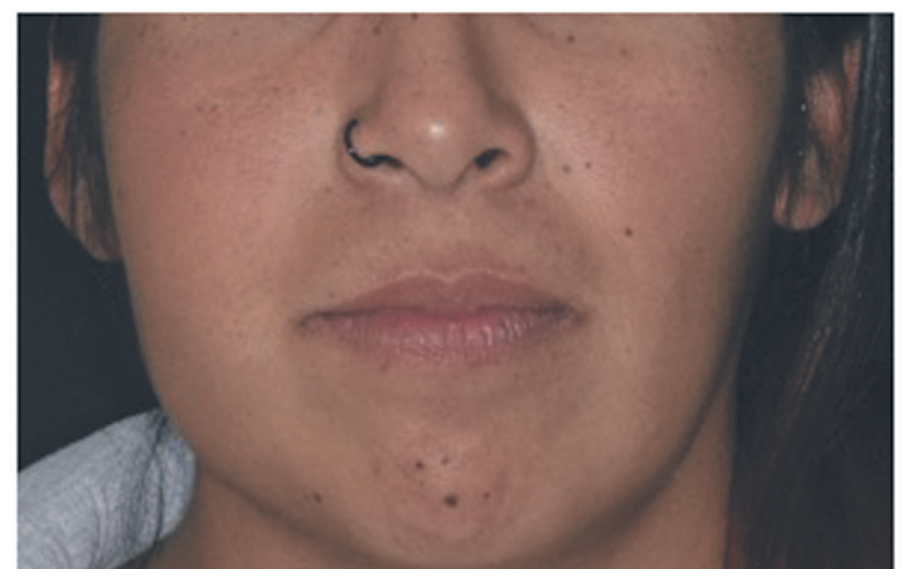

Figura 1. Examen Facial. Asimetría hemifacial derecha; moderado aumento de volumen en región de cuerpo y ángulo mandibular derecho.

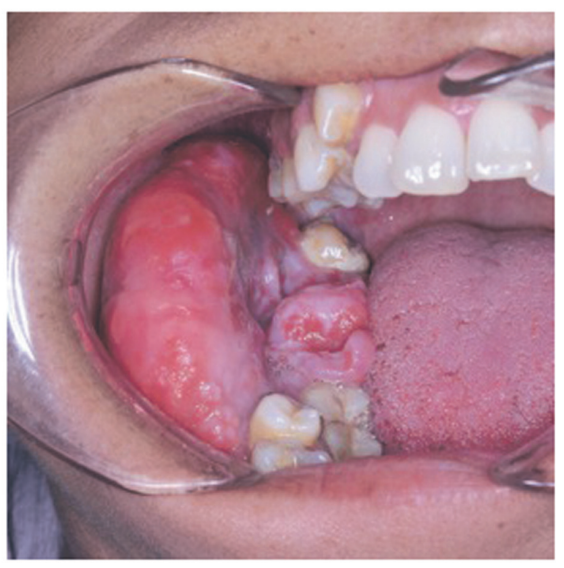

Figura 2. Examen Bucal. Lesión tumoral de considerables dimensiones que abarca cuerpo, ángulo y rama mandibular derecha, de límites definidos, consistencia firme, asociada a dientes desplazados y cuya mucosa que la recubre con aspecto fibroso como consecuencia del trauma oclusal.

Inicialmente se solicitó una radiografía panorámica (Fig. 3), exámenes de laboratorio y una Tomografía Computarizada (TC) con contraste. En la TC se observó una lesión de densidad mixta, de bordes definidos y corticalizados, con expansión de ambas tablas óseas, en la zona de cuerpo, ángulo y rama mandibular derecha. Sus dimensiones fueron $5.3 \times 3.3 \times 4.2 \mathrm{~cm}$ en los ejes sagital, transversal y cráneocaudal respectivamente. Destaca el aspecto anterior hipodenso y posterior de densidad mixta, con formación de tabiques óseos, junto con la presencia de los dientes 17 y 18 desplazados (Fig. 4).

Con los antecedentes clínicos y radiográficos obtenidos, se consideraron dos hipótesis diagnósticas, Ameloblastoma y Mixoma Odontogénico. La segunda etapa fue una biopsia incisional, en distintos sectores del tumor, obteniendo muestras de tejido blando extraóseo de consistencia firme y muestras de tejido duro lesional intraóseo. En el resultado de la histopatología se observó proliferación de tejido fibroblástico, con fibroblastos de núcleos alargados, cromatínicos, que se disponen en fascículos estrellados, y entre ellos con islotes

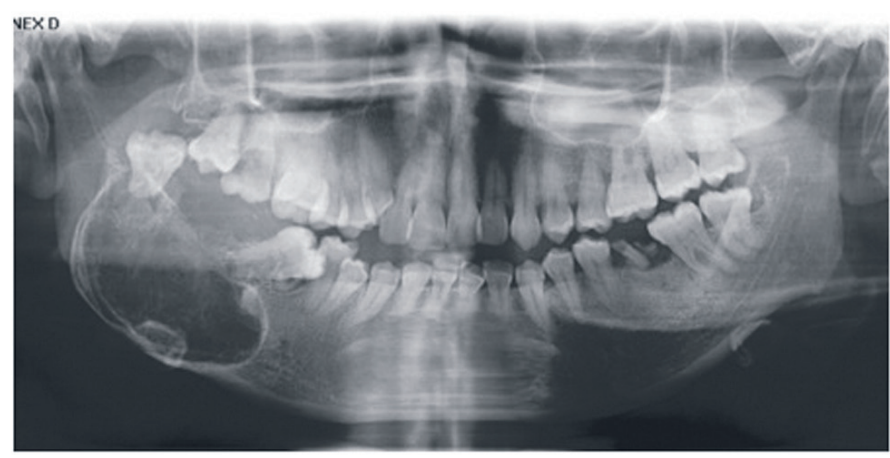

Figura 3. Radiografía Panorámica. Se observa extensa lesión de radiolucidez mixta y bordes corticalizados que compromete región de cuerpo, ángulo y rama mandibular derecha en relación a dientes desplazados.
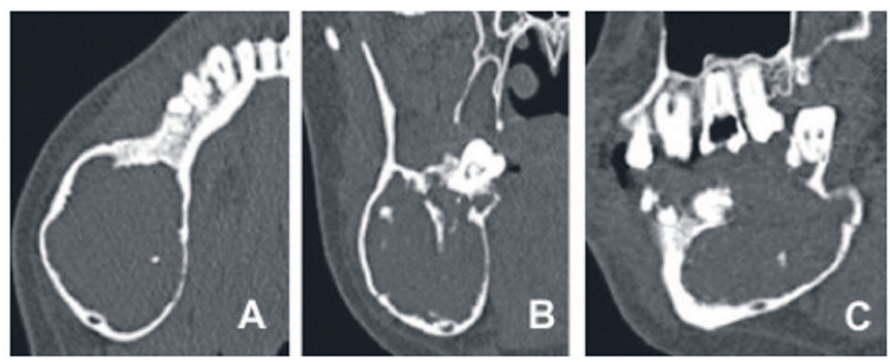

Figura 4. Tomografía Computarizada Prequirúrgica. Destaca el aspecto anterior hipodenso y posterior de densidad mixta, con formación de tabiques óseos. En los diferentes cortes (A. Axial, B. Coronal y C. Sagital) se puede ver expansión de las corticales óseas y desplazamiento inferior del nervio alveolar.
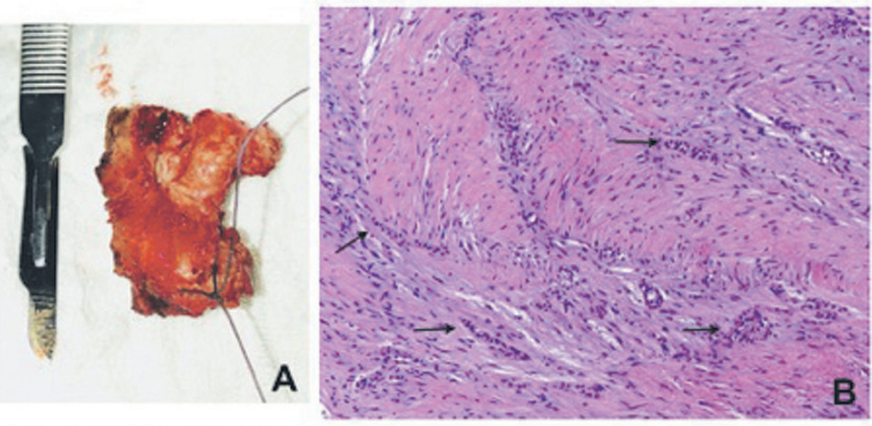

Figura 5. A. Muestra de tejido biopsiado B. Microfotografía de corte histológico tinción hemotoxilina-eosina $\times 100$. Se observa tejido conjuntivo con fibras colágenas y numerosos fibroblastos polimorfos, e islotes de epitelio odontogénico (flechas).

y cordones de epitelio odontogénico (Fig. 5). El resultado del informe histopatológico fue Fibroma Odontogénico. El plan de tratamiento se llevó a cabo bajo anestesia general. La técnica quirúrgica se realizó mediante un abordaje bucal amplio lineal en relación al límite tumoral paracortical realizándose la exéresis del segmento tumoral exofítico que comprometía la cavidad bucal, esta porción tenía alrededor de $2 \mathrm{~cm}$ de alto por $4 \mathrm{~cm}$ de largo y $2 \mathrm{~cm}$ de ancho (Fig. 6), luego se eliminó la masa tumoral intraóseo por segmentos, preservando las corticales medial, lateral y el piso de la lesión. Finalmente se procedió a síntesis por primera intención de la herida operatoria. No se consideró la instalación de una placa de osteosíntesis ya que la cavidad residual estaba delimitada por tablas óseas compactas, sin embargo, se instalaron tornillos de fijación intermaxilar en caso de presentarse necesidad de bloqueo intermaxilar postquirúrgico. No se presentaron complicaciones intraoperatorias, pero en los controles postoperatorios se produjo una dehiscencia de la herida quirúrgica, se indicaron lavados periódicos con clorhexidina y control semanal, promoviendo un cierre por segunda intención. En cuanto al manejo en el intraoperatorio del nervio mandibular, no hubo complicaciones de tipo quirúrgicas ya que éste se encontraba desplazado hacia el borde mandibular inferior, sin visualizarse durante la cirugía, sin embargo la 


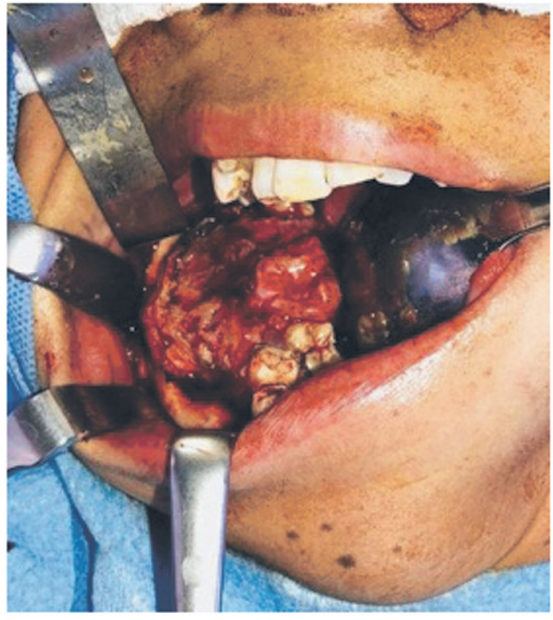

Figura 6. Abordaje bucal amplio para posterior enucleación de la lesión tumoral.

paciente cursó con una hipoestesia transitoria que cedió a las cuatro semanas.

Tras 11 meses de seguimiento postoperatorio la paciente persiste con una asimetría perimandibular derecha, producto de la deformación mandibular consecutiva a la expansión tumoral que en un segundo acto quirúrgico será corregida. Pero clínicamente no se ha evidenciado signos de recidiva (Fig. 7). Y en los controles radiográficos mediante TC se ha observado regeneración ósea de caudal a cefálico (Fig. 8).
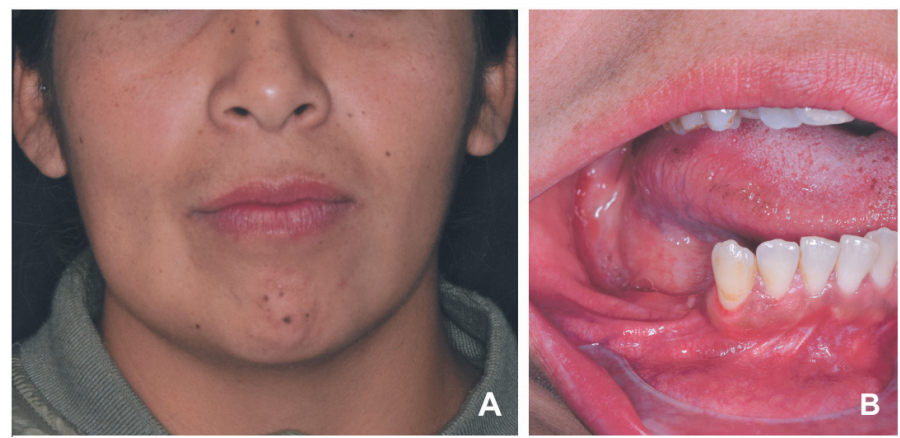

Figura 7. Control Clínico Postoperatorio (11 meses). A) Examen Facial Persistencia de aumento de volumen perimandibular derecho. B) Examen Bucal. Ausencia de lesión tumoral preexistente. Mucosa bucal indemne.
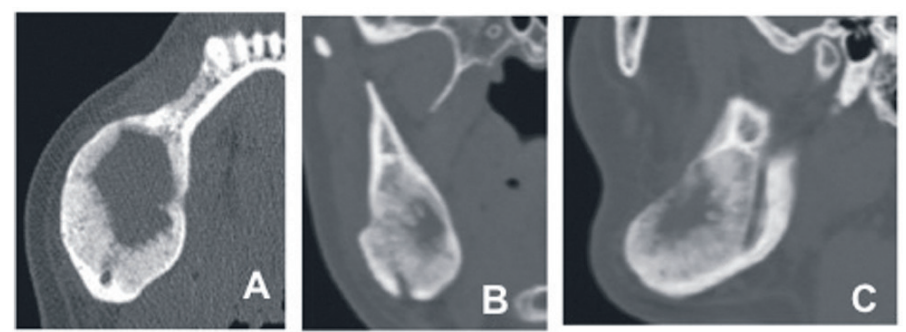

Figura 8. Tomografía Computarizada Postquirúrgica (8 meses). En los distintos cortes (A. Axial, B. Coronal y C. Sagital) se observa regeneración ósea de caudal a cefálico.

\section{DISCUSIÓN}

EI FOC es una neoplasia rara y benigna que puede confundirse con lesiones endodónticas y/o tumores odontogénicos ${ }^{(7)}$. Shafer et al. en 1983, describieron que el FOC era una neoplasia con características clínicas e histopatológicas diferentes al resto de los tumores odontogénicos ${ }^{(8)}$

Wesley et al. En 1975 sugirieron un conjunto de criterios diagnósticos para el FOC, entre los cuales encontramos ${ }^{(9)}$ :

1. Corresponde a un tumor que se presenta exclusivamente en los huesos maxilares, que posee un crecimiento lento y persistente produciendo expansión cortical.
2. Radiográficamente tiene apariencia variable, pero su presentación más frecuente corresponde a lesiones radiolúcidas multiloculares que involucran porciones relativamente grandes de los maxilares en etapas avanzadas. Además, en algunos casos lo podemos encontrar asociado a dientes no erupcionados y/o desplazados.

3. Histopatológicamente, su característica más importante es la presencia de fibras de colágeno maduras con numerosos fibroblastos intercalados. La presencia de pequeños nidos de epitelio odontogénico inactivo es una característica variable.

4. Corresponde a una lesión benigna y que responde bien a la enucleación quirúrgica sin tendencia a sufrir una transformación maligna.

Gardner en 1980 intentó clasificar histológicamente el FOC en tres tipos de lesiones diferentes, aunque probablemente relacionadas ${ }^{(5)}$.

1. Tipo folículo dental hiperplásico.

2. Tipo simple: neoplasia con tejido conjuntivo fibroso variable que contiene nidos de epitelio odontogénico simple.

3. Tipo WHO: lesión más compleja con características de dentina displásica o tejido similar al cemento y una cantidad variable de epitelio odontogénico. Debido a que este grupo era similar al tumor odontogénico calcificante descrito por Pindborg en 1971, Gardner lo designó como FOC tipo WHO. La característica distintiva entre las dos lesiones son que el tumor de Pindborg se tiñe con tinciones de amiloide.

Histológicamente, el tipo simple se caracteriza por una masa tumoral formada por fibras de colágeno maduras intercaladas generalmente por muchos fibroblastos uniformes en su ubicación y equidistantes entre sí. Además, puede presentar de manera variable pequeños nidos de epitelio odontogénico inactivos. El tipo WHO también consiste en tejido conectivo fibroso relativamente maduro pero bastante celular con pocas a muchas islas de epitelio odontogénico. Osteoide, dentina displásica o material similar al cemento también presente de manera variable(10).

Según Marx, el FOC requiere inicialmente una biopsia incisional porque su presentación sugiere una enfermedad más agresiva que puede confundirse con otras lesiones tumorales. El tratamiento de elección para el FOC es la enucleación y curetaje. La lesión tumoral se separa fácilmente de su cavidad ósea y no muestra evidencia de infiltración ósea. Su recurrencia se considera infrecuente en el contexto de las recidivas ${ }^{(11)}$. Dunlap y Barker presentaron dos casos de fibroma odontogénico maxilar tratados con legrado con un seguimiento de 9 años y 10 años sin evidencia de recurrencia ${ }^{(12)}$. Sin embargo, se han reportado recidivas. Heimdal et al. Informaron un caso que recurrió 9 años después de la cirugía(13). Svirsky et al. Han reportado una tasa de recurrencia del 13\% (2 de 15 casos) $)^{(14)}$. Jones et al. Informaron un caso que se repitió 16 meses después de la cirugía( ${ }^{(15)}$. Según Marx, si se observa recurrencia, se debe revisar la muestra patológica original así como la muestra de biopsia actual(11). Es posible que un mixoma odontogénico con características fibrosas a menudo denominado fibromixoma se haya interpretado como un FOC. También es posible que un fibroma osificante con pocos componentes óseos o similares al cemento se haya interpretado como un FOC.

\section{CONCLUSIONES}

Para el diagnóstico adecuado de FOC es importante tener en consideración todos los aspectos clínicos, imagenológicas e histopatológicos de esta lesión.

Debido a que el FOC es una patología rara, suele confundirse con otras lesiones de los maxilares, por lo que es importante considerarla dentro del diagnóstico diferencial.

Existen pocos casos clínicos de FOC a nivel Latinoamericano, por ende es indispensable realizar más reportes para poder establecer una casuística certera en la población.

\section{FUENTES DE FINANCIAMIENTO}

Este trabajo no cuenta con financiamiento alguno.

\section{CONFLICTO DE INTERESES}

Los autores declaran que no tienen ningún conflicto de intereses. 


\section{Bibliografía}

1. Philipsen $\mathrm{H}$, Reichart $\mathrm{P}$. Classification of odontogenic tumours. A historical review. J. Oral Pathol. Med. 2006;35(9):525-529.

2. Buchner A, Merrell P, Carpenter W. Relative frequency of central odontogenic tumors: A study of 1088 cases from Northern California and comparison to studies from other parts of the world. J Oral Maxillofac Surg. 2006;64:1343-52.

3. Ikeshima A, Utsunomiya T. Case report of intra-osseous fibroma: a study on odontogenic and desmoplastic fibromas with a review of the literature. J Oral Sci. 2005;47:149-57.

4. Muñoz TC, Reyes OP, Alvarez NC, Venegas RB. Fibroma odontogénico central, tipo WHO: Reporte de un caso y revisión de la literatura. Rev Esp Cir Oral Maxilofac. 2010; 32(4):159-164.

5. Gardner DG. The central odontogenic fibroma: an attempt at clarification. Oral Surg Oral Med Oral Pathol Oral Radiol Endod. 1980;50:425-32.

6. Kaffe I, Buchner A. Radiologic features of central odontogenic fibroma. Oral Surg Oral Med Oral Pathol. 1994:78:811-8.

7. Dunlap CL. Odontogenic fibroma. Semin Diagn Pathol. 1999;16:293-6.

8. Shafer WG, Hine MK, Levy BM. A textbook of oral pathology. 4th ed. Philadelphia: Saunders; 1983. pp. 263, 294-5.
9. Wesley RK, Wysocki GP, Mintz SM. The central odontogenic fibroma. Clinical and morphological studies. Oral Surg Oral Med Oral Pathol Oral Radiol Endod. 1975; 40:235-45.

10. Shafer WG, Hine MK, Levy BM. Shafer's textbook of oral pathology. 6th ed. Elsevier; 2009. pp. 294-5.

11. Marx RE. Diane stern, oral and maxillofacial pathology: A rationale for diagnosis and treatment. Quintessence Publishing; 2007. pp. 672-4.

12. Dunlap CL, Barker BF. Central odontogenic fibroma of the WHO type. Oral Surg Oral Med Oral Pathol Oral Radiol Endod. 1984;57:390-4.

13. Heimdal A, Isaacson G, Nilsson L. Recurrent odontogenic fibroma. Oral Surg Oral Med Oral Pathol Oral Radiol Endod. 1980;50:140-5.

14. Svirsky JA, Abbey LM, Kaugars GE. A clinical review of central odontogenic fibroma: With addition of 3 new cases. J Oral Med. 1986;41:51-4.

15. Jones GM, Eveson JW, Shepherd JP. Central odontogenic fibroma. A report of two controversial cases illustrating diagnostic dilemmas. Br J Oral Maxillofac Surg. 1989;27:406-11.

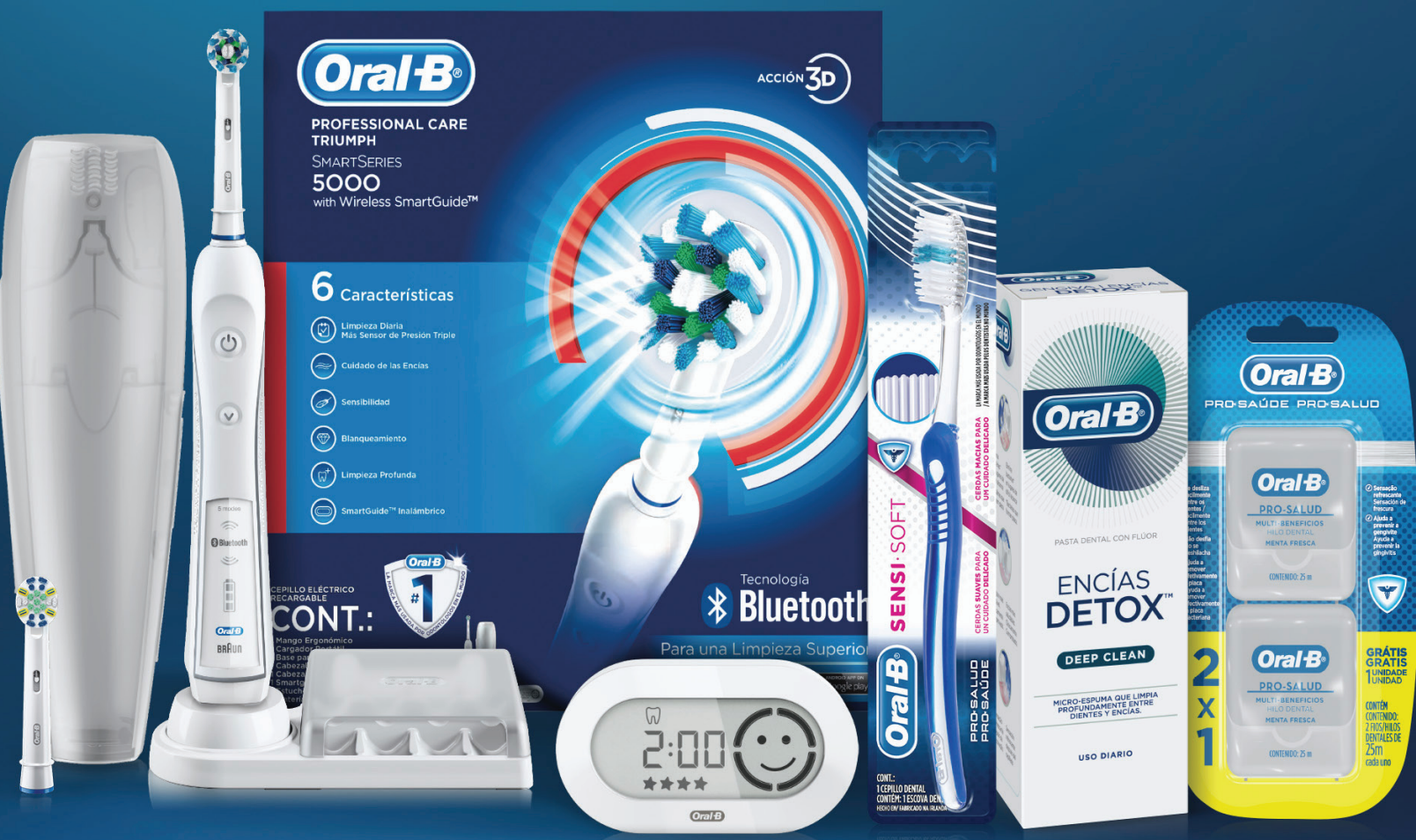

